\title{
La technologie au service des personnes âgées
}

Luigi Corrado

La gérontechnologie a pour but d'adapter des solutions innovantes aux problèmes de santé des personnes âgées. De nombreux produits existent aujourd'hui sur le marché qui peuvent faciliter la vie de nos aînés.

\section{Technologie für Senioren}

Ziel der sogenannten Gerontechnologie ist es, innovative Lösungen auf die Gesundheitsprobleme älterer Menschen abzustimmen. Auf dem Markt sind gegenwärtig zahlreiche Produkte zu finden, die Senioren das Leben erleichtern können.

DOI 10.1024/2297-5160/a000039

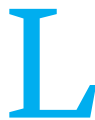
e vieillissement de la population est un défi sociétal majeur, où les structures dédiées au maintien à domicile vont jouer un rôle de plus en plus important.

\section{Technologie pour la vie quotidienne}

L'ampleur des enjeux qui pèsent aujourd'hui sur la question du vieillissement de la population et de l'augmentation du nombre de personnes âgées en perte d'autonomie suscite depuis quelques années une extraordinaire créativité dans le domaine des technologies au service de la santé et du bien-être des personnes âgées (la gérontechnologie).

La gérontechnologie a pour objectif de développer des produits, des services et des technologies appliquées aux besoins de la vie quotidienne des personnes âgées. Il s'agit d'adapter pour ces dernières des solutions innovantes aux problèmes de santé, d'habitat, de mobilité et de communication qu'elles rencontrent. Bien entendu la gérontechnologie comprend des domaines complexes comme la télémédecine ou la robotique, mais également des solutions simples et pouvant faciliter la vie de tous les jours des â̂nés. Pour cet article, nous avons choisi de vous présenter trois objets commercialisés et disponibles à des coûts abordables.

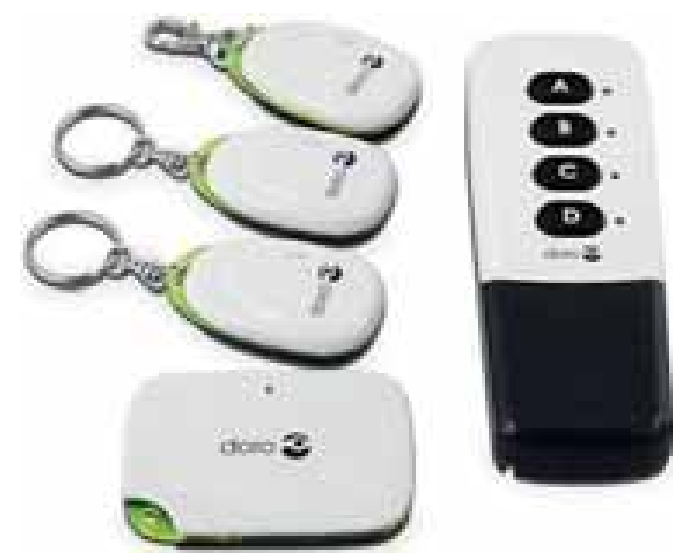

Doro Memory plus 335

\section{Détecteur de clé avec bip sonore}

Ce détecteur de la société Doro, qui développe des produits et applications pour les personnes âgées, permet à celles souffrant des troubles amnésiques de retrouver facilement leurs clés. Cet appareil comprend quatre détecteurs. Trois porte-clés, qui peuvent être fixés à des objets importants tels que les clés ou une sacoche. Le quatrième, au format d'une carte de crédit, pourrait par exemple se glisser dans un porte-monnaie. Le boîtier de recherche qui a l'apparence d'une télécommande est pourvu de quatre boutons. Lorsqu'un objet que l'on recherche est introuvable, il suffit d'appuyer sur la touche qui correspond pour provoquer un signal sonore et retrouver l'objet égaré. Lorsque la personne oublie de remettre le boîtier à sa place habituelle, ce dernier transmet un bip. La portée maximale entre les détecteurs et le boîtier de recherche est de 30 mètres.

\section{Indicateur de sonnerie avec amplificateur sonore}

Cet article de la société Geemarc est destiné à amplifier le son de la sonnette d'un appartement. Il est constitué d'un boîtier de réception amplificateur et d'une sonnette à bouton. Le boîtier de réception est déclenché à distance par la sonnette d'appel et permet à des personnes malentendantes éloignées de leur téléphone ou de leur sonnette 


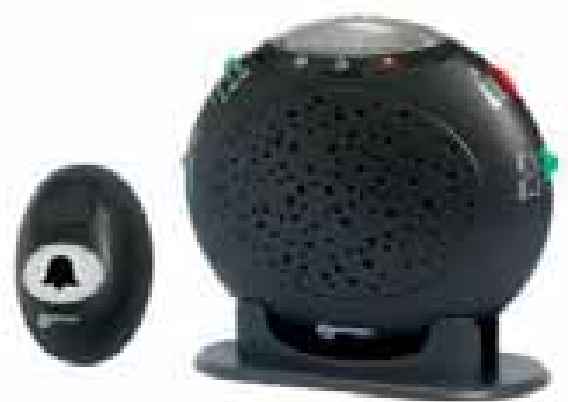

Geemarc Amplicall 20

d'entendre la sonnerie. Lorsqu'un appel retentit, le boîtier émet un signal sonore ou un signal lumineux, voire les deux en même temps, selon les réglages effectués au départ. Le niveau sonore du signal est réglable simplement et il permet le choix de trois tonalités de sonneries. Les sonneries émises par le boîtier ne sont pas les mêmes s'il s'agit d'un appel téléphonique ou de la sonnette. Ce système est conçu pour être utilisé à domicile, sa portée est de $15 \mathrm{~m}$ environ en habitation et le niveau sonore maximum est très élevé $>95 \mathrm{~dB}$ selon le fabricant. D'autres produits existent également comme le système Lisa.

\section{Ouvre tout}

Pour répondre à des troubles de la préhension musculaire, un manque de force même momentané ou encore aux personnes n'utilisant qu'une seule main, il existe actuellement plusieurs produits sur le marché. Nous avons choisi de retenir pour cet article l'ouvre-bocal One Touch. Le pro-

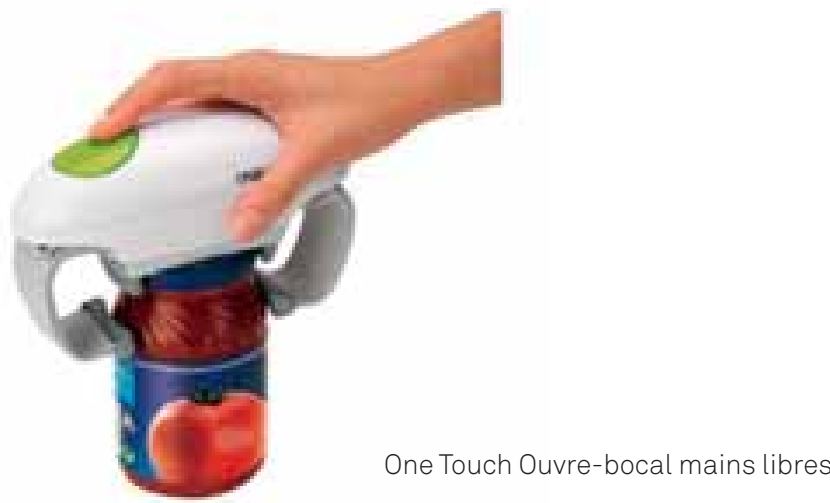

duit illustré sur l'image permet en le plaçant sur un bocal de l'ouvrir en appuyant sur le bouton vert. Son fonctionnement automatique facilite le quotidien et l'autonomie d'une personne âgée qui n'aura pas forcément besoin d'aide pour accomplir des actes simples de la vie quotidienne. Cet ouvre-bocal automatique s'utilise sur les bocaux ou bouchons ayant un couvercle de diamètre compris entre 3 et $10 \mathrm{~cm}$. De nombreux autres dispositifs manuels ou automatiques permettent de faciliter l'ouverture de bouteilles ou de boîtes.

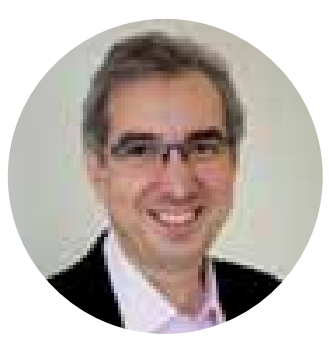

Luigi Corrado est directeur des Services généraux au sein de Institution genevoise de maintien à domicile (imad) à Genève (www.imad-ge.ch). Il préside le groupe de travail sur les Gérontechnologies de la Société Suisse de Gérontologie.

luigi.corrado@imad-ge.ch 